\title{
The Survey of Image Generation from EEG Signals based on Deep Learning
}

\author{
Delong Yang \\ Shenzhen Institute of Advanced \\ Technology Chinese Academy of \\ Sciences \\ yangdl@siat.ac.cn
}

\author{
Dongnan Su \\ Shenzhen Institute of Advanced \\ Technology Chinese Academy of \\ Sciences \\ dn.su@siat.ac.cn
}

\author{
Zhaohui Luo \\ Shenzhen Institute of Advanced \\ Technology Chinese Academy of \\ Sciences \\ luozh@siat.ac.cn
}

\author{
Peng Shang* \\ Shenzhen Institute of Advanced \\ Technology Chinese Academy of \\ Sciences \\ peng.shang@siat.ac.cn
}

\author{
Zhigang $\mathrm{Hu}$ \\ Henan University of Science and \\ Technology, hu.Robert @126.com
}

\begin{abstract}
China has become a high-risk region of stroke. Most patients with stroke suffer regular bouts of post-stroke limb dyskinesia. Nowadays, there isn't an effective treatment for these patients. Brain computer interface (BCI) establishes a new pathway to connect human brains and device, which provide an innovation method to repair the human brain nervous systems through rehabilitation training. However, one of the mainly brain activity recordings, Electroencephalogram (EEG), cannot be represented accurately by other algorithms. With the development of deep learning techniques, the topic of EEG signals' representation by image generation technique has become an important research area. This paper we introduced the basic concepts of BCI systems first, then we give a survey of image generation techniques from EEG signals. At last, we proposed an experimental scheme of dataset establishment which is used for post-stroke patients with upper limb dyskinesia
\end{abstract}

\section{KEYWORDS}

Brain computer interface (BCI), Electroencephalograph (EEG), Image generation, Deep learning

\section{ACM Reference Format:}

Delong Yang, Dongnan Su, Zhaohui Luo, Peng Shang*, and Zhigang Hu. 2021. The Survey of Image Generation from EEG Signals based on Deep Learning. In 2021 International Symposium on Biomedical Engineering and Computational Biology (BECB 2021), August 13-15, 2021, Nanchang, China. ACM, New York, NY, USA, 5 pages. https://doi.org/10.1145/3502060.3502151

\section{INTRODUCTION}

With the acceleration of aging population and the popularity of the unhealthy lifestyles of inhabitants, the stroke has an explosive growth trend in China, and most patients with stroke suffer from limb dyskinesia which seriously affect the patients' survival quality

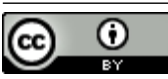

This work is licensed under a Creative Commons Attribution International 4.0 License.

BECB 2021, August 13-15, 2021, Nanchang, China

(C) 2021 Copyright held by the owner/author(s).

ACM ISBN 978-1-4503-8411-7/21/08.

https://doi.org/10.1145/3502060.3502151
[1]. Unfortunately, there are no therapeutic schedules can fully cure the disease. Nowadays, rehabilitation training is an effective method to improve the patients' prognosis and quality of life. However, rehabilitation physicians are insufficient and the passive treatments of post-stroke limb dyskinesia are not benefit to the brains' nervous systems, which restrict the effectiveness of rehabilitation training [2].

Active rehabilitation training requires the patients play an active role in the training program. It not only reduces the doctors' workload, but also benefits to the repair of injury in patients' nervous system. One of the most challenges during the active rehabilitation training is how to establish the bridge between the disabled limbs of the post-stroke patients and their brains. Hence, brain-computer interface (BCI) [3] has been chosen to read human mind. BCI is a technology which to realize the information propagation from human brains to external devices.

Electroencephalograph (EEG) [4] is the recording of scalp's electrical field which is produced by the human's brain activation. The electrical field originates from post-synaptic potentials of pyramidal neurons in the cerebral cortex. EEG is a neuroimaging technique and it is widely used in different area of clinical research, for example, sleep disorders and epilepsy. Moreover, due to the advantages of EEG such as higher time resolution that reach millisecond timescales, safety of use, portability and so on, EEG is becoming the principle brain signal of brain-computer interface (BCI) which is extremely important to active rehabilitation robot and healthcare.

However, there are several limitations which have been hinder the clinical application of EEG. First, some brain tissues such as skin, skull and meninges cause interference to the electric fields' transmission which are between the sources and sensors, resulting in low spatial resolution of EEG. Second, the brain activity is a complicated process, multiple physiological and environmental noise would disrupt the EEG signal. Finally, EEG is not a stationary signal and it varies with the individual. Above disadvantages make the EEG signals are difficult to recognize and distinguish.

Previous researches [5] have claimed that the EEG recordings contain visual information, even though the relationship between brain activation and human visual cortex is still unclear [6]. Hence, image generation technology from EEG [7] is a feasible solution for EEG signal analysis. Moreover, deep neural networks (DNNS) have 
already applied in different computer vision tasks, it also applies to EEG signal processing program.

In this paper, we first introduce the clinical application of BCI systems for post-stroke patients, then we summarize the research status of the relationship between image generation and EEG signals. In order to establish the dataset for DNNs, at last, we propose a clinical study conceptual to collect post-stroke patients' training sample.

\section{MATERIALS AND METHODS}

This section consisted of three parts, the introduction of BCI systems, research status for the modal exchanges of image and EEG and the clinical study conceptual for the special dataset establishment.

\subsection{The Introduction of BCI systems}

$\mathrm{BCI}$ system is a technology which establishes the bridge between human brains and external devices. It provides a new pathway for patients with post-stroke limb dyskinesia to communicate with environments without using the natural neuromuscular [8]. For instance, rehabilitation robots and intelligent wheelchairs for paralysis patients, speech translation systems for deaf people, artificial retinas for blindness [9]. Popular brain signals mainly include EEG, Electrocorticography (ECoG), Magnetoencephalography (MEG), Positro Emission Tomography (PET), functional Magnetic Resonance Imaging (fMRI), functional Near-Infrared Spectroscopy (fNIRS) and so on. Several major factors such as spatial resolution, time resolution, portability, safety of use and movement tolerability should be taken care of whether BCI systems can be applied in clinical treatments. To have an integrative consideration, EEG has a good trade-off among the above aspects.

Up to now, most achieved devices are invasive BCI systems. More unfortunately, all the BCI systems for patients are still remain in the laboratory stage, and they cannot be applied in clinical application and the patients' daily lives. However, with consideration of the surgical risks, patients' acceptability and conveniences, noninvasive $\mathrm{BCI}$ systems are the future trends. Based on the former fact, the clinical use of BCI systems have come a long way.

In recent years, several non-invasive BCI demos are shown in laboratories. A technology [10] which can translate human brain neural activity into speech is proposed in 2019. The kinematic and sound manifestations of human cortical activity are decoded to synthesize audible voice. The most famous noninvasive BCI system is the continuous neural tracking technique for mechanical arm [8]. This system allows the human subject to control a seven degrees of freedom mechanical arm to track a 2D moving target on a computer screen continuously.

In spite of the difficulties, researches of the noninvasive BCI systems have made evolutional breakthroughs. For some simple binary classify applications including the presence or absence of P300 detection [11] and seizure detection [12]. Due to the advantages of EEG, the BCI-robots which are based on EEG are future development directions.

\subsection{The Survey of image generation from EEG signals}

Previous discussions show that the EEG-based BCI systems have many merits, however, several defects are not avoided. The EEG signals are not stationary and vulnerable to noise interference. Moreover, they also vary from person to person. Those disadvantages cause EEG signal analysis to be a great challenge. In order to solve the above problems, use image generation technique to represent EEG signals has become one of research hot spots on EEG signal analysis.

Compared with waveform signals, images are more intuitive, meaningful, inspiring and complex. Due to the development of deep learning techniques, image generation technique is an innovation approach for EEG signals' representation.

Image and EEG signal are two different model data which cannot be translated in traditional methods. Due to deep learning technique, especially the generative adversarial networks, some bridges between these two models has been established preliminarily. Even though characteristics of the EEG signals which are non-stationarity, low signal-to-noise ratio, differ across people and ethical considerations cause some difference, EEG signals are also a kind of structured data to some degree.

EEG signals are composed mainly of Alpha wave $(8 \sim 15 \mathrm{~Hz})$, beta wave $(12.5 \sim 28 \mathrm{~Hz})$, theta wave $(4 \sim 7 \mathrm{~Hz})$ and delta wave $(1 \sim 4$ $\mathrm{Hz}$ ). The distribution and frequency of these waveforms are highly relevant to different human brain activities. In addition, there is some highly relationships between the expressions of deep learning models and the human brain representations [13]. The hierarchical layer structures of deep neural networks are similar to the visual representation of human brains [14]. Although this finding is still in infancy, it provides a new pathway to reverse engineered the human's brain activity [15]. The main technical character of deep learning is big data analytics. Even though there are several difficulties compared with computer vision area, to deduce human intentions via the deep learning models is possible. Recently, establishing the relationships between the images and EEG signals has become a practical solution which can be decode the EEG signals intuitively.

It is known that the percentage of human brain's visual cortex in total cortical area reaches about $30 \%$ [16], therefore the representation of visual information in human brains is a complicated procedure. As a result, an EEG signal respond to the special given visual stimuli $[17,18]$ and generate a special corresponding wave form which can be decoded from EEG signals to images.

Better understands of how to visualize the EEG signals which are generated by $\mathrm{CNN}$ layers and how to apply CNNs to decode the EEG signals have been displayed in deep learning with $\mathrm{CNN}$ for EEG decoding and visualization. Different deep learning architectures are studied to decode the images from the raw EEG signals. The first work to establish the bridge between the EEG signals and the visual object classifiers [19] was proposed in 2017. The authors design two deep networks which are a recurrent neural network to learn EEG data and a convolutional neural network to regress images to translate human brain process to image. The EEG signals and images are two different data schemas, how to learn meaningful features of the two modalities is significant important. 
Therefore, GAN is widely used for image generation. In general, a GAN is composed of a generator which is translating EEG signals to images and a discriminator which is judge the generated images are true or false. For example, [7] uses a LSTM RNN to design the discriminator and constructs the generator based on a conditional GAN. During testing, the generated images are similar to the input images. Professor He [20] proposes a semi-supervised cross-modal which is designed based on GAN to generate images form the brain EEG signals. Then, similar works are put forward successively in [21].

The deep learning models to generalize images form EEG data has gradually been a popular issue recently. It is feasible in theory to generate images from EEG signals because of a fact that the primary visual cortex is composed of organized oriented edge and color detectors. Outside information must through areas V4 [22] of the human brain which has larger receptive fields before they reach the inferior temporal cortex [23]. The development of deep learning technologies and neuroscience [24] makes the human-level visual representations for computer can be achieved.

As mentioned above, even though the bridge between the EEG signals and images has been established, this translation is inaccurate. As a result, we need further improvements such as specific classifiers or exact descriptions to get accurate outputs as far as possible when the images are translated from the EEG signals. Otherwise, the converse is not true because the EEG signals are more complicated than images.

Indeed, the information procession of the human brains is very complicated, we also face some problems such as how to interpret the exact meaning of the acquired data, what the concrete transformation mode between different modalities.

Moreover, many methods which are unrelated to visual understanding have been proposed. For example, [25] used CNNs to learn the EEG signals which is evoked by music and the accuracy of the song detector is increased by $28 \%$. A cognitive load classifier based on EEG representation is proposed in [26], the use of RNN and CNN makes the accuracy of the classifier reaches to about $90 \%$. Comparing with image generation techniques from the EEG signals, these non-visual applications have initially applied in real life practice. In clinical applications, seizure detection algorithms using CNN or RNN are proposed in [27] and [28], which are the successful performance of the EEG signals' application. The above methods act on no more than ten categories classifying and most of them can only apply to single BCI paradigm. Apparently, the generalization capabilities of them are suspected due to the special stimuli and corresponding task. For speech reconstruction task, [29] presents a new approach to reconstruct the speech stimuli which is affected by some noisy, but the procedure is invasive which cannot be widely promoted.

\subsection{The Clinical Study Conceptual for Dataset Establishment}

A deep neural network is composed of multiple layers, the information processing program of the DNN extracts high-level features from prior layers to next layers, which is similar to human brains' thinking progress to a certain degree. However, human nervous systems are born of years of learning, a DNN has a large number of parameters which are unknown. As a result, design and implementation of deep learning models cannot do without the support of datasets. Several successful applications in deep learning are all carried out based on the related datasets, such as face detection, object recognition and self-driving technology. However, comparing with computer vision algorithms, EEG datasets are obviously insufficient.

The subjects contain healthy adults which form the control group and post-stroke patients with upper limb dyskinesia which form the experimental group. All the subjects aged between 40 to 70 , data collection is under way currently. This work is supported by Dapeng New District Nan'ao People's Hospital and Shenzhen Second People's Hospital. We have tried various experimental schemes, for example, the subjects under eyes-open and eyes-close states respectively, the subjects lie flat and sit respectively, the subjects image denoted posture actively or do the same postures based on the researchers' direction.

The subjects' thoughts fly apart when they under the eyes-close state (the corresponding EEG signal can be shown in Figure 1 ), the subjects easily fall into slumber when they lie flat, the subjects cannot image denoted posture accurately. After many times experiments, we determine the eyes-open state, sit and external guidance as the final experimental scheme, as shown in Figure 2.

There are 31 hand postures according to our statistics, but only 6 hand postures are needed for post-stroke patients under the clinical doctors' instruction. The 6 hand postures are grasp, open the palm, finger oppositions between thumb and other fingers. Therefore, we only record the EEG signals which are corresponding to above postures. The time consuming of each posture is spread over 5 seconds and the time interval between two consecutive postures is about 10 seconds. All the hand postures occur in a random fashion. The hand postures and corresponding EEG signals are recorded simultaneously, as a sample of the dataset.

Regretfully, the dataset for post-stroke patients with upper limb dyskinesia had not been complicated, hence, we can only give an experimental explanation in this paper. In the future, we will opensource data for public after the work is done.

\section{CONCLUSION}

Neural activity (recorded by EEG) and visual data have very different structures, and finding a common representation is not trivial. Brain analysis is the most complicated cognitive process which is still unclear. Both neural and computer scientists are trying to explain the detail procedure. However, all the plausible strategies for brain activity explanation have not been proved. The image generation technique based on deep learning is an innovative approach to represent EEG signals and this technique has already obtained some achievements. This paper sums up current researches of the image generation from EEG signals which are based on deep learning, then give an experimental scheme for the dataset establishment of "hand-motion and corresponding EEG signals". In the future, with the devices' development and the related datasets become abundant, we believe that EEG signals can be represented through images.

\section{ACKNOWLEDGMENTS}

This work was supported in part by the Guangdong Province Science and Technology Program Grant (2018A050501010), SIAT Innovation Program for Excellent Young Researchers (E1G062), the 


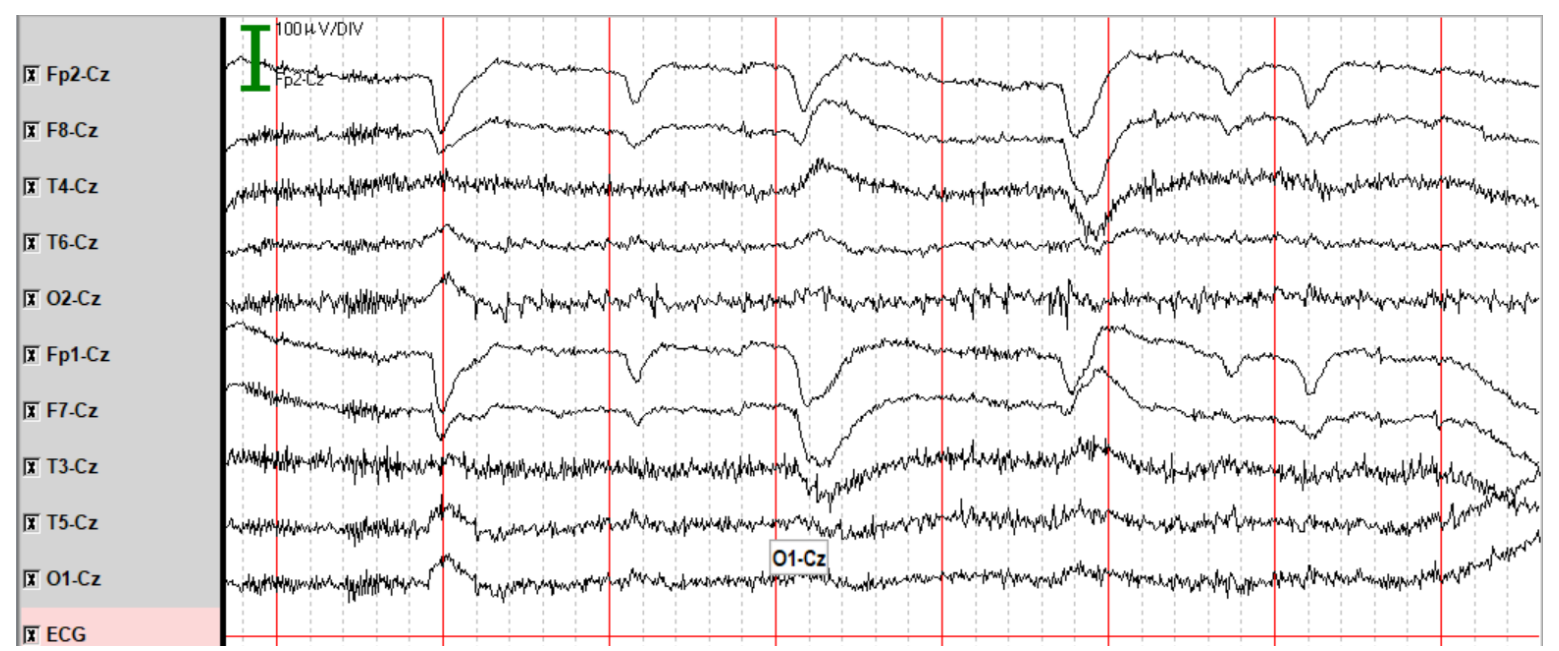

Figure 1: The corresponding EEG signals when the subject under the eye-close state.

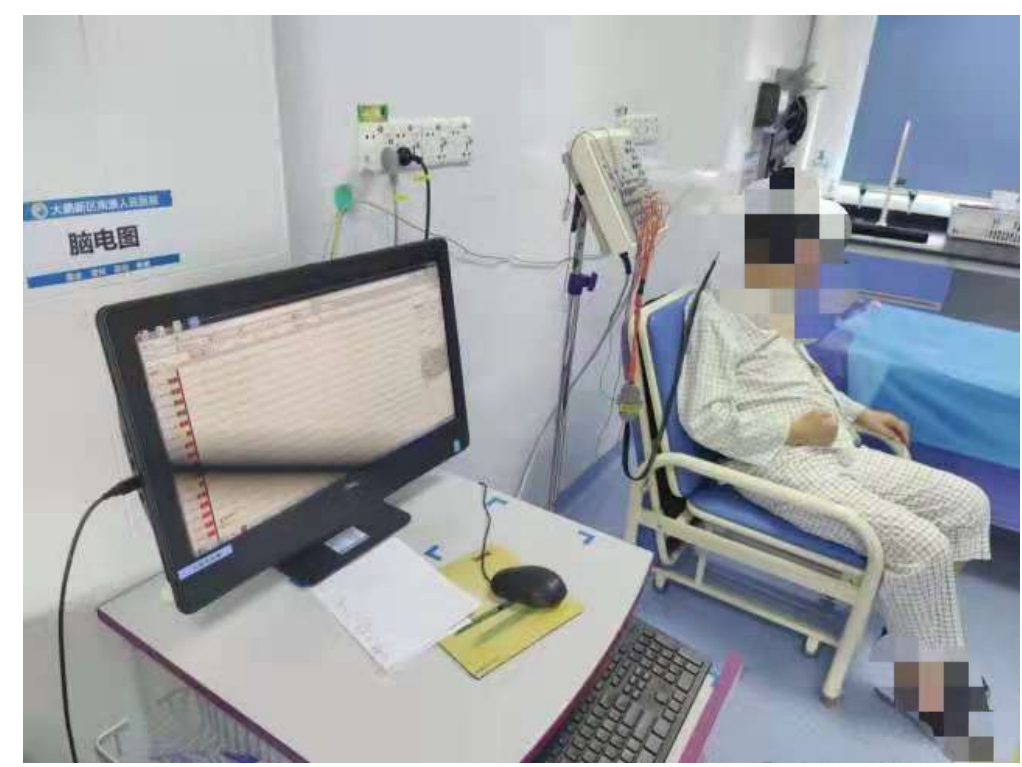

Figure 2: The final data collection progress of post-stroke patients with upper limb dyskinesia.

Shenzhen Governmental Science and Technology for Basic Research Program Grant (JCYJ20180507182446643), and the Shenzhen Science and Technology for International Cooperation Research Program Grant (GJHZ20190821155201661).

\section{REFERENCES}

[1] Guoyuan Yang, Kunling Jin, Zhijun Zhang, 2019. Methodology of Experimental Stroke Model. Shanghai Jiao Tong University Press.

[2] Cheng N , Phua K S , Lai H S , et al. 2020 Brain-Computer Interface-Based Soft Robotic Glove Rehabilitation for Stroke, J. IEEE Transactions on Biomedical Engineering, 2020, PP(99):1-1.

[3] Lebedev, Mikhail A., et al. 2006 Brain-machine interface:past present and future, J. Trends in Neurosciences, 2006, 29(9),536-546.

[4] Yanglan, Yu, Bin, et al. 2019 The Relationship between Biological Motion-Based Visual Consciousness and Attention: An Electroencephalograph Study - ScienceDirect, J. Neuroscience 415(C):230-240.

[5] C. Wang, S. Xiong, X. Hu, L. Yao, and J. Zhang. 2012. Combining features from ERP components in single-trial EEG for discriminating four-category visual objects. J Neural Eng, 9(5):056013, Oct 2012

[6] M. V. Peelen and P. E. Downing. 2007.The neural basis of visual body perception. Nat. Rev. Neurosci., 8(8):636-648, Aug 2007.

[7] Palazzo S , Spampinato C, Kavasidis I , et al. 2017. Generative Adversarial Networks Conditioned by Brain Signals. In Proceedings of the IEEE International Conference on Computer Vision (ICCV). IEEE, 2017.

[8] Edelman, B.J.,Meng, J. et al. 2019. Noninvasive neuroimaging enhances continuous neural tracking for robotic device control[J], Science Robotics, 31(4),1-13.

[9] Makin JG, Moses DA, et al. 2020. Machine translation of cortical activity to text with an encoder-decoder framework[J], Nature Neuroscience, 23(4),575-582.

[10] Anumanchipalli G K, Chartier J , Chang E F. 2019. Speech synthesis from neural decoding of spoken sentences[J]. Nature, 568(7753):493-498.

[11] H. Cecotti and A. Graser. 2011. Convolutional neural networks for p300 detection with application to brain-computer interfaces. IEEE Transactions on Pattern Analysis and Machine Intelligence, 33(3):433-445

[12] P. Mirowski, D. Madhavan, Y. Lecun, and R. Kuzniecky 2009. Classification of patterns of EEG synchronization for seizure prediction. Clin Neurophysiol, 120(11):1927-1940 
[13] R. M. Cichy, A. Khosla, D. Pantazis, A. Torralba, and A. Oliva, 2016. Comparison of deep neural networks to spatio-temporal cortical dynamics of human visual object recognition reveals hierarchical correspondence. Sci Rep, vol. 6, p. 27755 , 06

[14] T. Horikawa and Y. Kamitani, 2017. Generic decoding of seen and imagined objects using hierarchical visual features. Nat Commun, vol. 8, p. 15037

[15] D. E. Stansbury, T. Naselaris, and J. L. Gallant, 2013. Natural scene statistics account for the representation of scene categories in human visual cortex. Neuron, vol. 79 , no. 5 , pp. $1025-1034$

[16] D. Grady, 1993. The vision thing: Mainly in the brain. Discover, 14(6):56-66

[17] H. Cecotti and A. Graser, 2011. Convolutional neural networks for p300 detection with application to brain-computer interfaces. IEEE transactions on pattern analysis and machine intelligence, 33(3):433-445

[18] M. Nakanishi, Y. Wang, Y.-T. Wang, Y. Mitsukura, and T.-P. Jung. 2014. A highspeed brain speller using steady-state visual evoked potentials. International journal of neural systems, 24(06):1450019

[19] Spampinato C, Palazzo S , Kavasidis I , et al. 2017. Deep Learning Human Mind for Automated Visual Classification. In Proceedings of the IEEE Conference on Computer Vision and Pattern Recognition (CVPR). IEEE, 2017.

[20] D. Li, C. Du, H. He. 2020. Semi-supervised cross-modal image generation with generative adversarial networks. Pattern Recognit., 100(1), p.107085

[21] I. Kavasidis, S. Palazzo, C. Spampinato, D. Giordano, and M. Shah, 2017. Brain2image: Converting brain signals into images. in ACMMM '17, 2017, pp.
1809-1817.

[22] J. W. Peirce. 2015. Understanding mid-level representations in visual processing. J Vis, vol. 15 , no. 7 , p. 5

[23] C. P. Hung, G. Kreiman, T. Poggio, and J. J. DiCarlo. 2005. Fast readout of object identity from macaque inferior temporal cortex, Science, vol. 310, no. 5749, pp. 863-866

[24] T. Horikawa and Y. Kamitani. 2017. Generic decoding of seen and imagined objects using hierarchical visual features. Nat Commun, vol. 8, p. 15037

[25] S. Stober, A. Sternin, A. M. Owen, and J. A. Grahn. 2016. Deep feature learning for EEG recordings. In To appear on ICLR 2016.

[26] P. Bashivan, I. Rish, M. Yeasin, and N. Codella. 2016. Learning representations from EEG with deep recurrent-convolutional neural networks. In To appear on ICLR 2016.

[27] P. Yan, F. Wang, and Z. Grinspan. 2018. Spectrographic seizure detection using deep learning with convolutional neural networks (s19.004)," Neurology, vol. 90, no. 15 Supplement.

[28] L. Vidyaratne, A. Glandon, M. Alam, and K. M. Iftekharuddin. 2016. Deep recurrent neural network for seizure detection," in 2016 International Joint Conference on Neural Networks (IJCNN), pp.1202-1207.

[29] B. N. Pasley, S. V. David, N. Mesgarani, A. Flinker, S. A. Shamma, N. E. Crone, R. T. Knight, and E. F. Chang. 2012. Reconstructing speech from human auditory cortex. PLoS Biol, 10(1): e1001251 